\title{
Regulation of human intestinal T-cell responses by type 1 interferon-STAT1 signaling is disrupted in inflammatory bowel disease
}

\author{
EM Giles ${ }^{1,2}$, TJ Sanders ${ }^{3}$, NE McCarthy ${ }^{1}$, J Lung ${ }^{1}$, M Pathak $^{1}$, TT MacDonald ${ }^{1}$, JO Lindsay ${ }^{1}$ and AJ Stagg $^{1}$
}

Type 1 interferon (IFN-1) promotes regulatory T-cell function to suppress inflammation in the mouse intestine, but little is known about IFN-1 in the human gut. We therefore assessed the influence of IFN-1 on CD4 + T-cells isolated from human colon tissue obtained from healthy controls or patients with inflammatory bowel disease (IBD). Immunofluorescent imaging revealed constitutive expression of IFN $\beta$ in human intestinal tissue, and colonic T-cells were responsive to exogenous IFN-1 as assessed by phosphorylation of signal transduction and activator of transcription 1 (pSTAT1) and induction of interferon stimulated genes (ISGs). Unlike their blood counterparts, intestinal T-cells from non-inflamed regions of IBD colon displayed enhanced responsiveness to IFN-1, increased frequency of pSTAT1 + cells, and greater induction of ISGs upon IFN-1 exposure in vitro. In healthy tissue, antibody neutralization of IFN $\beta$ selectively reduced T-cell production of the pro-regulatory cytokine interleukin-10 (IL-10) and increased IFN $\gamma$ synthesis. In contrast, neutralization of IFN $\beta$ in IBD tissue cultures increased the frequency of T-cells producing inflammatory cytokines but did not alter IL-10 expression. These data support a role for endogenous IFN-1 as a context-dependent modulator of T-cell function that promotes regulatory activity in healthy human intestine, but indicate that the IFN-1/STAT1 pathway is dysregulated in inflammatory bowel disease.

\section{INTRODUCTION}

In the healthy intestine, regulatory mechanisms limit $\mathrm{T}$-cell responsiveness to the commensal microbiota and environmental antigens in order to maintain homeostasis, but dysregulation of these mechanisms can lead to pathological inflammation and development of inflammatory bowel disease (IBD). Accordingly, intestinal T-cells remain one of the main targets for IBD therapy, ${ }^{1}$ but the endogenous factors that regulate T-cell activity in the human gut are not fully understood.

The balance of T-cell regulatory and effector functions are determined partly during priming in gut-associated lymphoid tissue or mesenteric lymph nodes. Signals derived from the activating dendritic cell and local environment can influence the differentiation of naive CD4 + T-cells toward a variety of effector or regulatory fates. However, it is now clear that T-cell properties are not fixed during priming but instead these cells retain functional plasticity and their function can be modified by signals encountered later upon homing to the intestinal mucosa. ${ }^{2-4}$

Type 1 interferon (IFN-1), predominantly IFN $\alpha$ and IFN $\beta$, has emerged as a potent context/timing-dependent modifier of T-cell function. ${ }^{5}$ IFN-1 binds to the IFN-1 receptor (IFNAR) and can differentially activate members of the signal transduction and transcription (STAT) family to determine downstream cytokine production. ${ }^{6}$ For example, in murine T-cells IFN-1 signaling via STAT1 inhibits IFN $\gamma$ production, whereas signaling via STAT4 instead promotes IFN $\gamma$. $^{7}$

IFN-1 limits viral replication in acute infection, but can also contribute to viral persistence in chronic infection models via the induction of IL-10 producing CD4 + T-cells. This suggests a potential role for IFN-1 in chronic T-cell-driven inflammatory disorders. ${ }^{8-10}$ In mice, constitutive low-level production of IFN $\beta$ occurs in several tissues even in the absence of viral

${ }^{1}$ Department of Immunobiology, Blizard Institute, Barts and The London School of Medicine and Dentistry, Queen Mary, University of London, London, UK. ${ }^{2}$ Department of Paediatric Gastroenterology, Monash Children's Hospital, Melbourne, VIC, Australia and ${ }^{3}$ Sir William Dunn School of Pathology, University of Oxford, Oxford, UK. Correspondence: EM Giles (e.m.giles@qmul.ac.uk) 
infection. ${ }^{11}$ This suggests that, at least in mice, endogenous IFN1 may regulate T-cell expression of STAT proteins and control responsiveness to subsequent stimuli. ${ }^{6}$ In murine models, IFNAR deficiency increases susceptibility to dextran sodium sulfate colitis, ${ }^{12}$ whereas administration of IFN-1 can ameliorate dextran sodium sulfate colitis via a mechanism that depends on TLR9/bacterial DNA. ${ }^{13,14}$ However, reports that local delivery of IFN-1 by a transgenic Lactobacillus acidophilus led to increased severity of dextran sodium sulfate colitis ${ }^{12}$ underscore the complex role of IFN-1 in maintaining mucosal integrity.

In a T-cell transfer model of colitis, IFN-1 signaling induced by poly(I:C) can ameliorate gut inflammation via a mechanism thought to require both FoxP3 + regulatory T-cells ${ }^{15,16}$ and IFNAR expression by the grafted colitogenic T-cells. ${ }^{17}$ In human multiple sclerosis, therapeutic administration of IFN-1 promotes T-reg IL-10 expression while restricting IL-17-driven inflammation. ${ }^{18}$ While genome-wide association studies have detected IFNAR variants associated with increased risk for developing IBD, ${ }^{19}$ IFN-1 therapy displayed only limited efficacy in human IBD. ${ }^{20}$ Given that constitutive expression and function of IFN-1 in human intestinal immunity is not known, we aimed to determine how IFN-1 influences signaling pathways at single-cell resolution in defined populations of intestinal T-cells in health and IBD.

\section{RESULTS}

\section{IFN-1 is constitutively expressed in human colon and} modulates the function of intestinal T-cells

To assess whether IFN-1 influences T-cell function in the human intestine, we first sought evidence that $\mathrm{T}$-cells are responsive to IFN-1 in human colon. To do this, we assessed mRNA expression levels of interferon-stimulated genes (ISGs; myxovirus resistance $\mathrm{A}-M x A$ and $2^{\prime}-5^{\prime}$ oligoadenylate synthetase $-2^{\prime} 5^{\prime} O A S$ ) in cells migrated out of human colonic biopsy tissue during overnight incubation in the presence or absence of IFN-1. The intestinal T-cells were then isolated by fluorescence-activated cell sorting (FACS) and subjected to quantitative reverse transcriptase-PCR. Alternatively, cells were obtained without IFN-1, but then exposed or not to brief (15 min) IFN-1 stimulation, and then permeabilized for assessment of pSTAT1 expression. Both approaches revealed that IFN-1 treatment of either healthy colon or IBD tissue increased intestinal T-cell expression of $M x A$ (median 30-fold, Figure 1a) and 2'5'OAS (median 50-fold, Figure 1b), and induced an $\sim 10$-fold increase in the frequency of CD4+ T-cells expressing pSTAT1 (Figure 1d, representative FACS plots Figure 1c).

We next sought to identify direct evidence of IFN-1 in the human colon, focusing on the IFN $\beta$ isoform thought to coordinate many key effects of IFN- $1{ }^{6}$ Technical challenges have hampered previous attempts to directly detect IFN-1 in human tissues, but a recent publication successfully observed IFN $\beta$ expression in human skin using immunohistochemistry analysis of freshly frozen clinical samples. ${ }^{21}$ We therefore used a similar immunohistochemistry approach to visualize endogenous IFN $\beta$ in colonic tissue. IFN $\beta$ was detected, distributed in the epithelium and also throughout the lamina propria in close association with intestinal T-cells, in both controls and IBD patients (Figure 1e, $\mathrm{f}$ and see Supplementary Figure S1 online).

We next sought evidence that human intestinal T-cells can be influenced by IFN-1 exposure in situ. We detected PSTAT1 in both naïve and memory subsets of CD $4+$ T-cells both before and after IFN-1 stimulation, but the frequency of pSTAT1 + cells was significantly higher in the stimulated memory cell population (see Supplementary Figure S2). Accordingly, expression of the T-cell activation marker CD69 was detected only among CD45RA - memory cells, and the CD69 + population included a higher frequency of pSTAT1 + cells, suggesting increased responsiveness to IFN-1 specifically in the activated memory T-cell compartment (see Supplementary Figure S3). Since these cells exhibited low-level "constitutive" phosphorylation of STAT1 and expression of ISGs even in the absence of IFN-1 supplementation (Figure 1a-d), we next assessed whether exposure to endogenous IFN-1 during the cell isolation process was sufficient to induce STAT1 signaling. To do this, we established walk-out cultures in the presence or absence of neutralizing anti-IFN $\beta$ antibody and assessed T-cell expression of total STAT1 and pSTAT1. As phosphorylation is a brief process and the neutralization was conducted over the 24 -h walk-out period, it was felt more likely to detect an effect in total STAT1 expression, which is known to be influenced by IFN-1 signaling. ${ }^{6}$ Neutralization of IFN $\beta$ significantly reduced the frequency of U-STAT1 + $\mathrm{CD} 4+\mathrm{T}$-cells detected (Figure 2a and $\mathbf{b}$ ), suggesting that constitutively produced IFN $\beta$ was biologically active in these cultures. Additionally, these cells had reduced responsiveness to further IFN-1 stimulation with decreased frequency of pSTAT1 + cells (Figure $2 \mathbf{c}$, $\mathbf{d}$ ). This is consistent with endogenous IFN $\beta$ priming $\mathrm{CD} 4+\mathrm{T}$-cells in their responsiveness to IFN-1. In contrast, addition of neutralizing anti-IFN $\gamma$ did not significantly alter STAT1 phosphorylation or total STAT1 levels (data not shown). Collectively these data support a role for IFN $\beta$ produced constitutively within human intestinal tissue as a potential modulator of gut $\mathrm{T}$-cell function, signaling at least in part via STAT1 activation.

\section{Phosphorylation of STAT1 and IFN $\beta$ responsiveness are} enhanced in intestinal CD4 + T-cells from IBD patients

In order to assess whether IFN-1:STAT1 signaling is altered in human IBD, we next compared CD4 $+\mathrm{T}$-cell responsiveness with IFN $\beta$ and pSTAT1 expression between IBD patients and control donors. To do this, we focused our analyses on cells isolated from macroscopically and histologically non-inflamed areas of colon in order to exclude signaling signatures associated with active inflammation. Even in unstimulated tissue cultures, we observed marked expression of pSTAT1 among CD4 + T-cells isolated from the intestinal mucosa (Figure 1c, d), although the total frequency of pSTAT1 + cells was significantly higher in colonic tissue from IBD patients (Figure 1d). Upon supplementation with exogenous IFN-1, both IBD colon samples and control tissues exhibited $\sim 10$-fold 
a

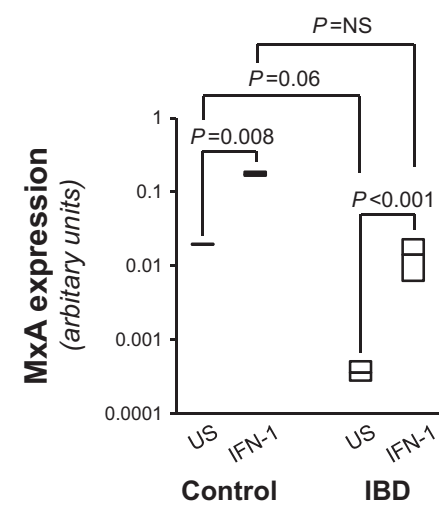

C
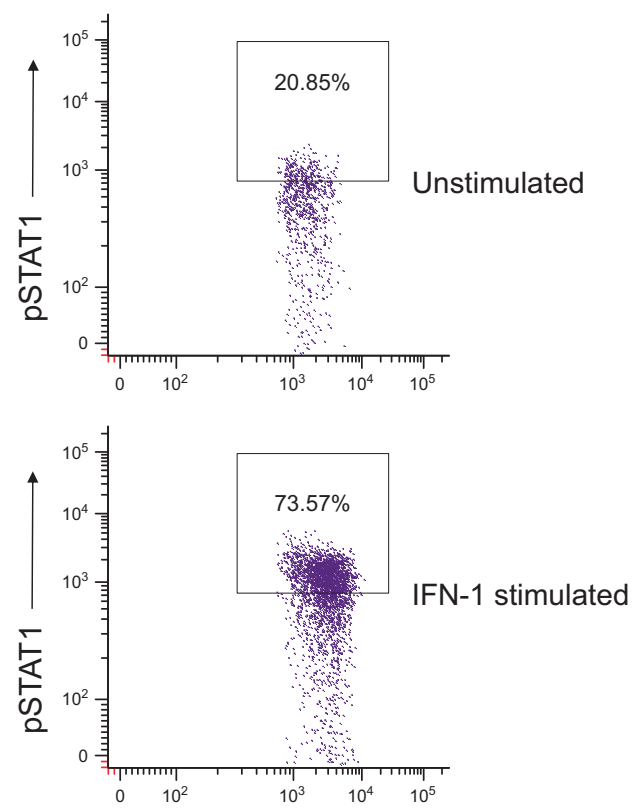

b

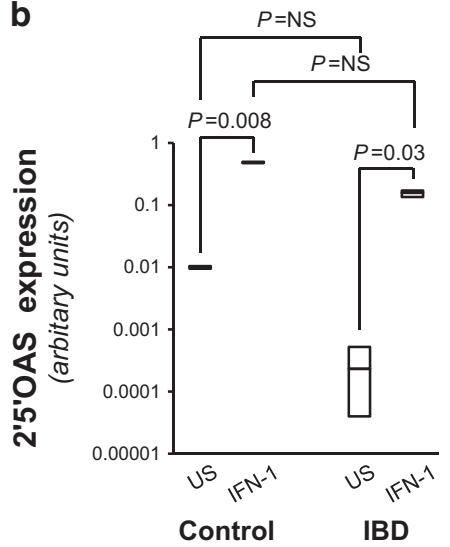

d

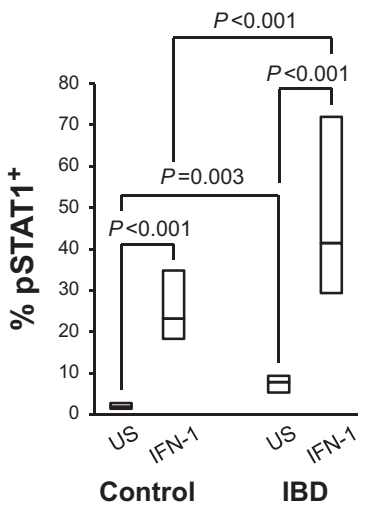

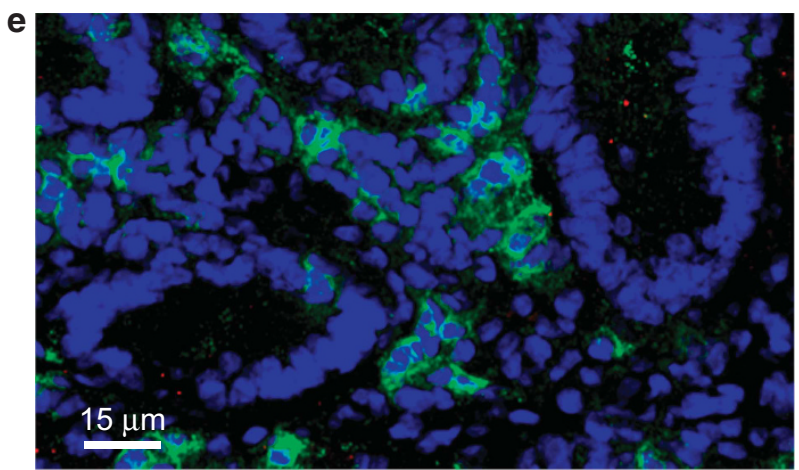

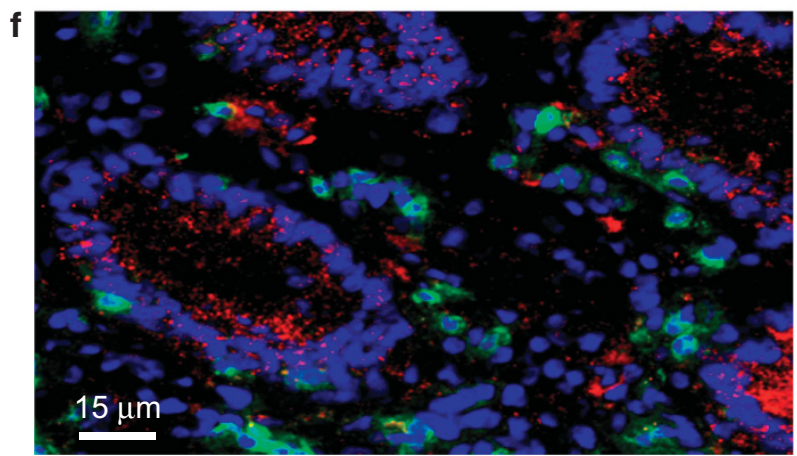

Figure 1 Human intestinal T-cells are responsive to type 1 interferon (IFN-1) in healthy and inflammatory bowel disease (IBD). (a, b) Intestinal samples were isolated from controls $(n=8)$ or IBD patients $(n=10)$ in the presence of IFN-1 for $24 \mathrm{~h}$ or unstimulated (US) and cells were sorted for CD3 + viable cells. RNA was extracted and expression of ISGs MxA (a) and $2^{\prime} 5^{\prime} \mathrm{OAS}$ (b) were measured by quantitative reverse transcriptase-PCR. (c) Representative flow cytometry plots of cells isolated by walk out. Cells were stimulated (lower panel) or not (upper panel) with IFN-1 for 15 min. (d) Combined results of controls $(n=16)$ and IBD patients $(n=24)$ showing frequency of pSTAT1 + CD4 + T-cells with IFN-1 stimulation or not (US). White boxes indicate first to third quartiles with middle line indicating median value. (e, f) Fresh frozen sections prepared from control colon tissue for immunohistochemistry. CD3 as green (AF488). (e) Isotype staining mlgG1 (AF555-red). (f) IFN 3 (AF555—red). Representative of five independent experiments.

increases in the proportion of derivative cells containing pSTAT1 (Figure 1d). Similar data were obtained in parallel analysis of samples of inflamed mucosal tissue from IBD patients, although the total proportion of pSTAT1 + cells detected was lower (see Supplementary Figure S4). These data indicated that under both resting and inflammatory conditions, CD4 + T-cells in IBD colon exhibit increased STAT1 activation compared with their counterparts in control tissue samples. We therefore proceeded to investigate whether the increased levels of pSTAT1 detected in CD4 + T-cells in IBD tissue could indicate enhanced Th1 polarization, as described in an earlier report. ${ }^{22}$ However, our analyses revealed that pSTAT1 was expressed at comparable levels in both Tbet + (Th1 cells) and Tbet - subsets of gut CD4 + T-cells (Figure 3a-c), indicating that pSTAT1 was not a surrogate marker of Th1 cells in this setting.

Collectively these data demonstrated that STAT1 phosphorylation and putative IFN-1 signaling were modulated in T-cells that migrated out of IBD colon, irrespective of whether the tissue was overtly inflamed. Conceptually, these effects could reflect intrinsic T-cell properties, exposure to soluble factors released by the cultured tissue, or a combination of these 

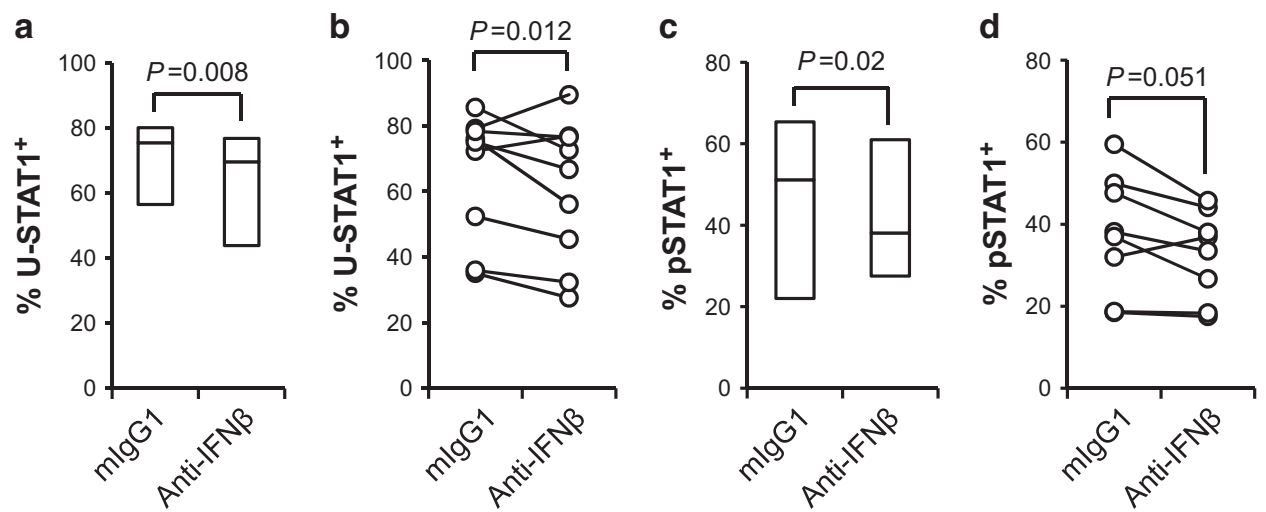

Figure 2 Endogenous IFN-1 supports U-STAT1 expression and IFN-1:pSTAT1 responsiveness in human intestinal CD4 + T-cells. (a-d) Lamina propria mononuclear cells were walked-out in the presence of anti-IFN $\beta$ antibody or isotype control. Cells were analyzed by flow cytometry gated on scatter and CD4 + . (a) Expression (total) (U-)STAT1 in CD4 + intestinal T-cells $(n=20)$. (b) As per (a), showing paired values from control samples only $(n=8)$. (c) pSTAT1 expression in CD4 + intestinal T-cells $(n=20)$. (d) As per c, showing paired values from control samples only $(n=8)$. IFN-1, type 1 interferon STAT1, signal transduction and activator of transcription 1.
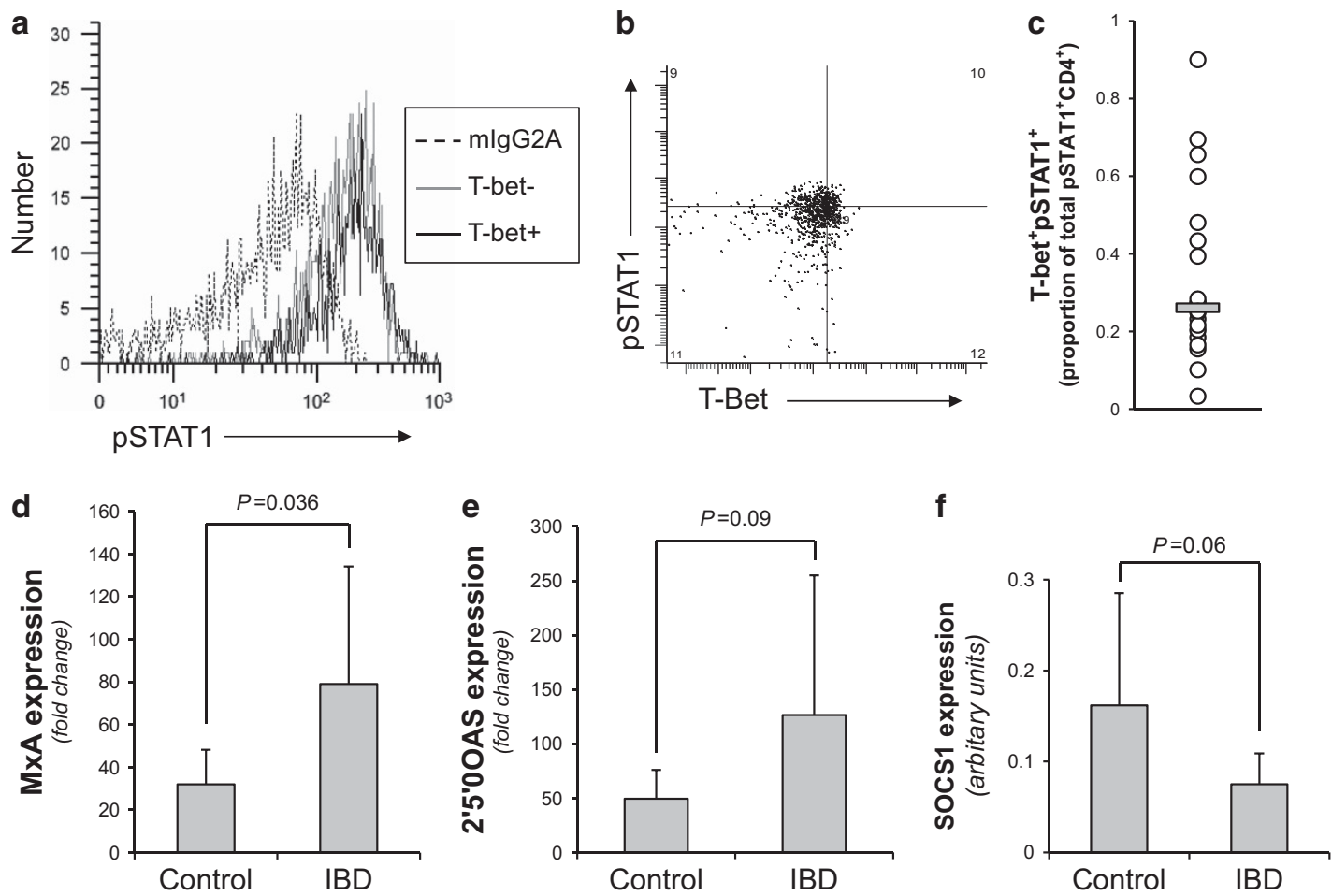

Figure 3 Intestinal T-cells are more responsive to type 1 interferon (IFN-1) in inflammatory bowel disease (IBD), not simply due to an increase in Th1 phenotype. Lamina propria mononuclear cells were isolated by walk-out and processed for phosflow (a-c) or intestinal T-cells were flow sorted and mRNA processed for quantitative reverse transcriptase-PCR (d-f). (a) Representative flow cytometry plot of pSTAT1 expression in T-bet + (black line) and T-bet ${ }^{-}$(gray line) gated on CD4 + T-cells (dotted line mlgG2A isotype). (b) Two-dimensional representative fluorescence-activated cell sorting plot of CD4 + T-cells showing co-expression of T-bet and pSTAT1. (c) Proportion of all T-Bet + CD4 + T-cells that are also pSTAT1 $+(n=23)$. Gray line indicates median. (d, e) Expression of ISGs MxA in d and 2' $5^{\prime} \mathrm{OAS}$ in e after $24 \mathrm{~h}$ walk-out cell isolation in the presence of IFN-1, comparing the samples with IFN-1 to those without in controls $(n=8)$ and IBD $(n=10)$ (f) Intestinal T-cells were isolated immediately by collagenase digestion before RNA extraction. Expression of SOCS1 from controls $(n=8)$ and IBD $(n=10)$. Bars indicate median expression with lines indicating standard deviation.

effects. To test for the presence of IBD-associated factors that might initiate STAT1 phosphorylation, we assessed the ability of IBD tissue culture supernatants to induce pSTAT1 in CD4 + T-cells derived from control colon tissue. Despite extensive investigation, we found no consistent evidence that soluble factors released from IBD tissue could upregulate pSTAT1 in healthy colonic CD4 + T-cells (data not shown). These findings indicated that IBD-associated changes in STAT1 phosphorylation and responsiveness to IFN-1 were at least in part due to intrinsic properties of the T-cells. Accordingly, we 
observed that gut T-cells from IBD patients exhibited greater IFN-1-induced ISG expression (Figure 3d, e). Furthermore, we detected a strong trend toward reduced expression of the STAT1 regulator SOCS1 (suppressor of cytokine signaling 1) in intestinal T-cells freshly isolated from IBD patients (Figure 3f). Taken together, these data indicate that altered IFN-1 responsiveness and STAT1 phosphorylation in IBD are at least in part due to changes in gut T-cell-intrinsic properties.

\section{IFN $\beta$ :STAT1 signaling exerts differential effects on T-cell function in healthy tissue and IBD colon}

When we assessed the ability of IFN-1 to activate alternative STAT family members in intestinal $\mathrm{CD} 4+\mathrm{T}$-cells, we observed marked phosphorylation of both STAT3 (twofold increase) and STAT5 (fourfold increase) in response to IFN-1 exposure, but we did not detect any differences between patients and controls either at baseline or in the cytokinetreated cultures (Figure 4a). These data suggested that differential effects of IFN $\beta$ on STAT activation in T-cells from IBD patients were specific to STAT1. We therefore proceeded to investigate whether the increased IFN-1 responsiveness and STAT1 phosphorylation observed in IBD patient T-cells was restricted to the gut or reflected system-wide changes in T-cell function. To do this, we analyzed CD4 + T-cells in peripheral blood and observed that the trace numbers of pSTAT1 + cells were significantly increased by IFN-1 stimulation, particularly within the CD45RA - memory/effector compartment $(32.1 \% \pm 22$ pSTAT1 + post stimulation $)$ in CD45RA - cells vs. $18.2 \% \pm 15.2$ in CD45RA + cells, $P<0.05$ ANOVA). However, we observed no significant difference in IFN $\beta$ responsiveness between IBD patients and controls even when comparing gut-homing $\mathrm{CD} 4+\mathrm{T}$-cells $(\beta 7$ integrin +$)$ with $\mathrm{CD} 4+\mathrm{T}$-cells targeted to other sites ( $\beta 7$-negative; Figure $4 \mathbf{b}$ ). These data indicated that IBD-associated changes in IFN-1 responsiveness and pSTAT1 expression of intestinal CD4 + T-cells are local effects that do not reflect systemic alterations in these patients.

There is increasing evidence from murine models that constitutive IFN $\beta$ expression can support regulatory T-cell responses, ${ }^{15,16}$ but the function of this cytokine in the human intestine is currently unknown. To address this question, we obtained T-cells from colonic biopsy tissue cultured in the presence or absence of neutralizing anti-IFN $\beta$ antibody and measured cytokine production and expression of master regulator transcription factors upon re-stimulation with anti-CD3 and anti-CD28. Addition of anti-IFN $\beta$ to the biopsy cultures did not alter T-cell expression of the transcription factors T-bet or FoxP3 as assessed by intracellular staining (Figure 5a, b), and ROR $\gamma$ t expression was very low throughout (data not shown). Nonetheless, intracellular cytokine staining of cells isolated from healthy tissue revealed that anti-IFN $\beta$ treatment induced a significant decrease in the frequency of $\mathrm{CD} 4+\mathrm{T}$-cells producing IL-10 (Figure 5c), with a trend towards an increased frequency of IFN $\gamma$-producing cells (Figure 5d), accompanied by a significant increase in supernatant concentration of IFN $\gamma$ detected by enzyme-linked
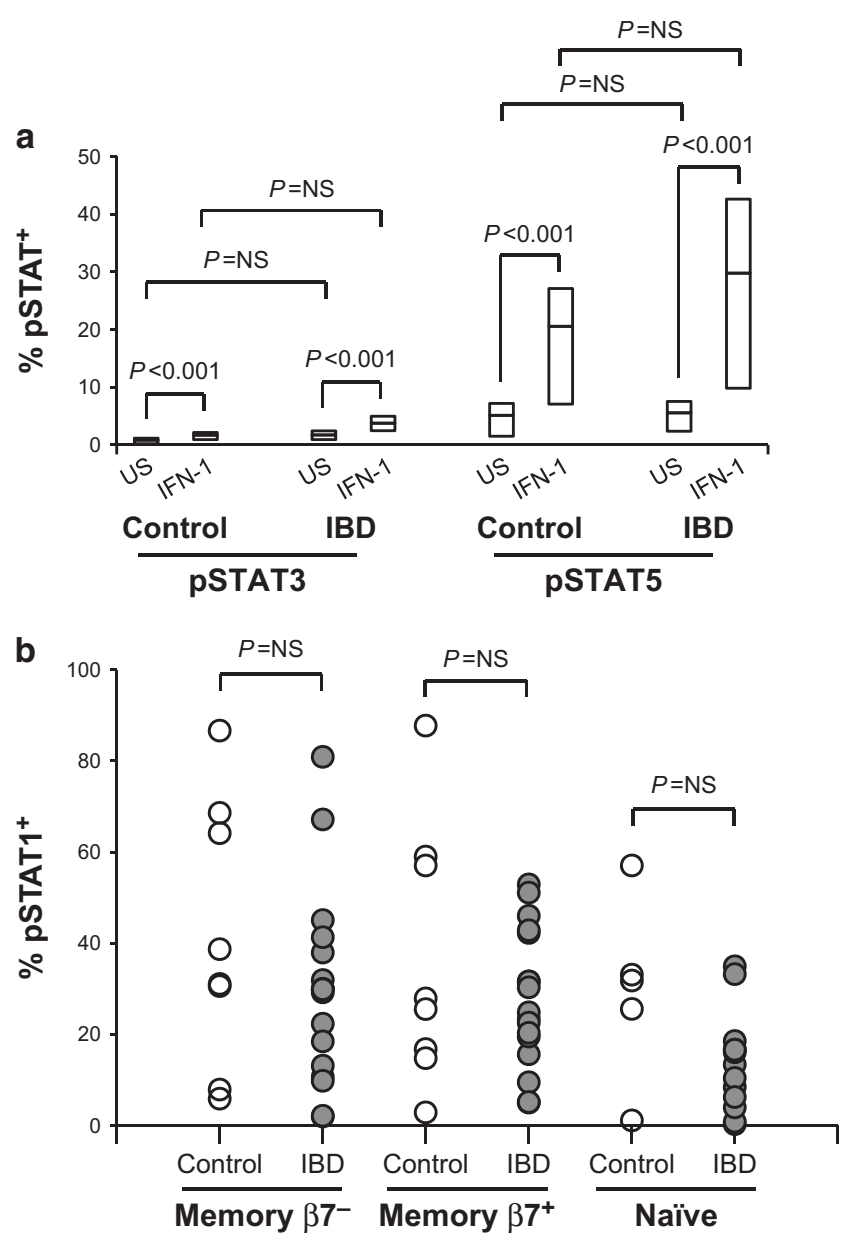

Figure 4 Inflammatory bowel disease (IBD)-associated alteration in STAT phosphorylation are specific for STAT1 and are not seen in the peripheral blood. (a) Walk-out cells were isolated from controls $(n=16)$ or IBD patients $(n=24)$, and either stimulated with type 1 interferon (IFN-1) for $15 \mathrm{~min}$ or not (US). The percentage of pSTAT3 + (left) or pSTAT5 + (right) CD4 + T-cells are shown. (b) Peripheral blood mononuclear cells were isolated from IBD patients $(n=18)$ and age-matched controls (8). Cells were analyzed for PSTAT1 signal after $15 \mathrm{~min}$ stimulation with IFN-1, depending on T-cell subset, $\mathrm{CD} 3^{+} \mathrm{CD} 4^{+} \mathrm{CD} 45 \mathrm{RA}^{-} \beta 7^{-}$(memory $\beta 7^{-}$), $\mathrm{CD}^{+} \mathrm{CD}^{+}{ }^{+} \mathrm{CD} 45 \mathrm{RA}^{-} \beta 7^{+}$(memory $\beta 7^{+}$) or $\mathrm{CD} 3^{+} \mathrm{CD} 4{ }^{+} \mathrm{CD}_{45 \mathrm{RA}}{ }^{+}$ (naive).

immunosorbent assay (ELISA) (Figure 6b). Tumor necrosis factor- $\alpha$ and IL-17A production were also observed, although IFN $\beta$ neutralization failed to alter the amount detected either by intracellular staining (Figures $5 \mathbf{e}, \mathbf{f}$ ) or by ELISA measurement (Figures $\mathbf{6 c}$, d). These findings suggest a potentially important role for endogenous IFN $\beta$ in modulating the balance of IFN $\gamma$ and IL-10 production by local T-cells in healthy tissues to favor the expression of anti-inflammatory cytokines. This effect is likely due to functional modulation by environmental signals rather than altered $\mathrm{T}$-cell differentiation fates, since the transcription factor profiles observed were not altered by IFN-1 exposure.

These "pro-regulatory" effects of IFN $\beta$ treatment in control tissues were absent when we repeated these experiments using colon samples from IBD patients, even though the tissues 

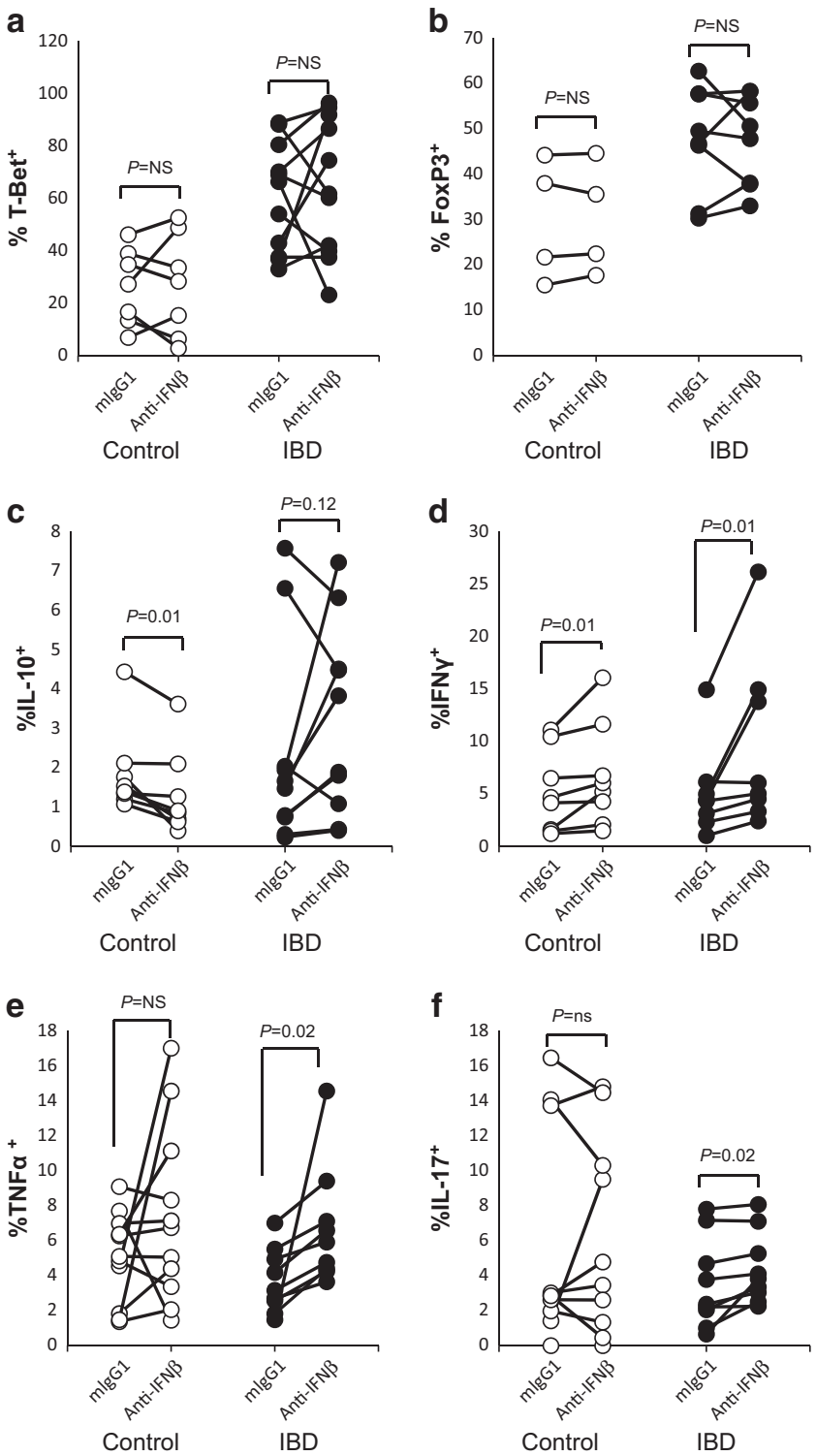

Figure 5 IFN $\beta$ neutralization decreases IL-10 production from intestinal CD4 + T-cells in healthy controls, but increases multiple cytokines in samples from non-inflamed inflammatory bowel disease (IBD) samples. Lamina propria mononuclear cells (LPMCs) were isolated in the presence of anti-IFN $\beta$ or isotype control (mlgG1). LPMCs were then collected and stimulated with CD3/CD28 antibodies for $24 \mathrm{~h}$. Intracellular cytokines or transcitpion factors were measured by flow cytometry as follows: (a) T-bet, (b) FoxP3, (c) IL-10, (d) IFN $\gamma$, (e) TNF $\alpha$, and (f) IL-17. Cells were gated on scatter and CD4 +. IBD, inflammatory bowel disease; IFN $\beta$, interferon beta; IL-10, interleukin-10; TNF $\alpha$, tumor necrosis factor- $\alpha$.

analyzed were obtained from non-inflamed regions of the intestine (macroscopically and histologically normal; Figures $\mathbf{5}$ and 6). In IBD biopsy organ culture, treatment with neutralizing anti-IFN $\beta$ induced an increase in the proportion of intestinal T-cells producing IFN $\gamma$, tumor necrosis factor$\alpha$, and IL-17A, and trend towards increased production of IL-10 (Figure 5a), accompanied by a significant increase in supernatant IL-10 levels as assessed by ELISA (Figure 6a). Consistent with previous reports, T-cells expressing T-bet or
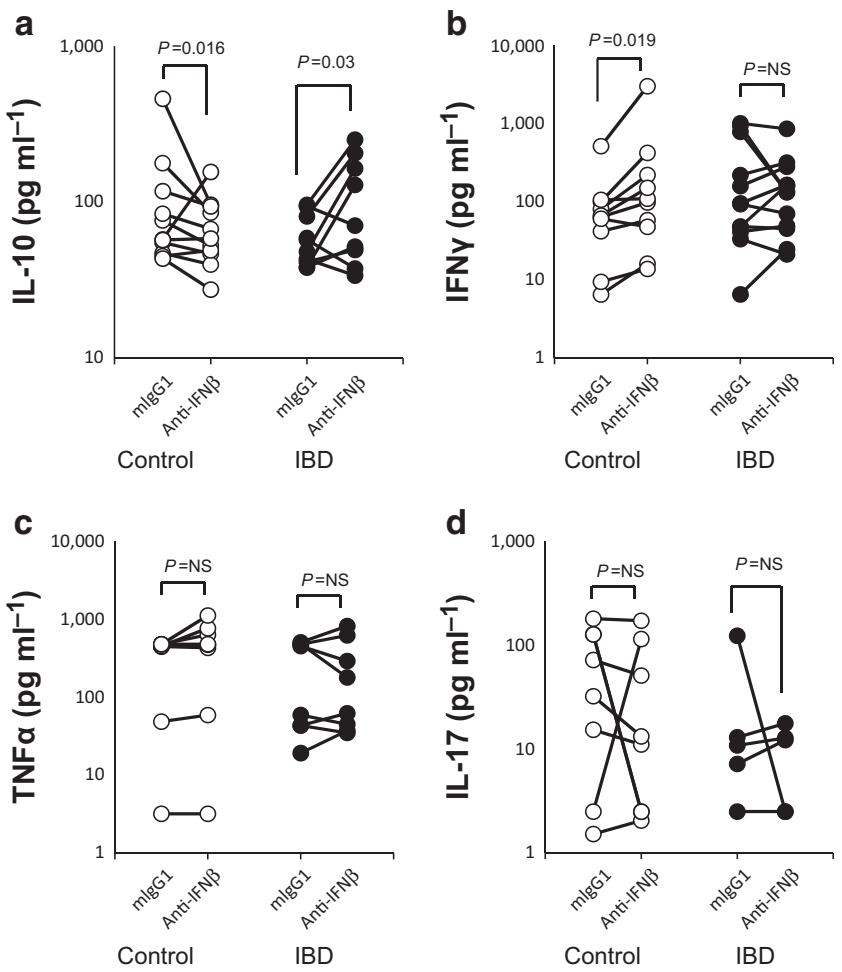

Figure 6 IFN $\beta$ neutralization increases IFN $\gamma$ concentrations in supernatants from control mucosa, but increased IL-10 concentrations from non-inflammatory bowel disease (IBD) mucosa. Cells were isolated in the presence of neutralizing anti-IFN $\beta$ antibody or isotype control (mlgG1). The supernatant was collected and IL-10 (a), IFN $\gamma$ (b), TNF $\alpha$ (c), IL-17 (d), and IFN $\alpha$ were measured by multiplex enzyme-linked immunosorbent assay ( $n=11$ controls, $n=11$ IBD). IFN $\beta$, interferon beta; IL-10, interleukin-10; TNF $\alpha$, tumor necrosis factor- $\alpha$.

Foxp3 were more frequent in IBD tissue than in control samples (see Supplementary Figure S5), ${ }^{23,24}$ but as observed in healthy tissue expression of these transcriptional regulators was not altered by neutralization of IFN $\beta$ (figures $\mathbf{5 a}, \mathbf{b}$ ). Therefore, endogenous IFN $\beta$ has a pro-regulatory effect in healthy tissue, where this cytokine promotes IL-10 production, whereas in IBD our data indicate that IFN $\beta$ instead restricts a number of effector $\mathrm{T}$-cells producing cytokines in the intestine.

\section{DISCUSSION}

This study reports that endogenous IFN $\beta$ promotes STAT1 phosphorylation in human intestinal T-cells to exert proregulatory effects in the healthy gut. We also present evidence that this axis is disrupted in IBD, where endogenous IFN $\beta$ instead restricts effector T-cell cytokine responses, even when assessing macroscopically and histologically non-inflamed areas of the gut.

We observed that T-cells isolated from human intestinal tissue were responsive to IFN-1 in vitro, as demonstrated by phosphorylation of multiple STAT signaling proteins and marked induction of ISG expression. The induction of ISG expression was on total $(\mathrm{CD} 3+) \mathrm{T}$-cells and the differential contribution of $\mathrm{CD} 8$ and $\mathrm{CD} 4$ populations cannot be determined. We also detected that a proportion of $\mathrm{T}$-cells 
migrating out of cultured biopsy tissue expressed the phosphorylated form of STAT1, which is the primary family member involved in IFN-1 signaling. This finding suggested that gut T-cells may be exposed to IFN $\beta$ cytokine in situ, and accordingly we observed for the first time that IFN $\beta$ is present in human colonic tissue. It is unclear what the source of the IFN-1 is although the epithelium appears to be important and this would be an interesting area for further research.

There is increasing evidence from murine models that constitutive production of IFN-1, and IFN $\beta$ in particular, can exert immunoregulatory effects. ${ }^{15,16,25}$ Consistent with this concept, neutralization of IFN $\beta$ in biopsy cultures from healthy donors significantly reduced the generation of IL-10 + T-cells, whereas the frequency of IFN $\gamma+$ T-cells and quantity of IFN $\gamma$ detected in the culture supernatants were increased.

The differences between the intracellular staining of the intestinal T-cells compared with the ELISA data may be due to the contribution of other cell types in the supernatants and their differential response to IFN $\beta$. It is likely that other IFN-1 subtypes are also present in the environment and it is not possible to conclude about the functional importance of all IFN-1 in this system. However, the overall findings are consistent with data from the murine T-cell transfer model of colitis, in which IFN-1 was found to be critical for maintaining regulatory T-cell function. ${ }^{15,16}$ However, in contrast with the experimental models, our data did not demonstrate any effect of IFN-1 on expression of FoxP3, which is less tightly linked to regulatory function in the human system. ${ }^{26}$

It has been suggested that the IFN-1 effects on T-cell function are context dependent. ${ }^{27}$ Consistent with this concept, our data demonstrate that the effects of IFN $\beta$ differ in healthy and IBD mucosa. Our findings are best explained by plasticity of intestinal T-cells and their responsiveness to local environmental signals. Our analysis provided no evidence for altered differentiation to major T-cell fates as defined by expression of master transcriptional regulators.

Phosphorylation of STAT1 in gut T-cells was demonstrated within $15 \mathrm{~min}$ of exposure to IFN-1, suggesting a direct effect of IFN-1 on T-cells, but it is not possible to exclude a contribution from other cell types in these cultures to many of the effects measured. The varied results obtained by ELISA analysis of supernatants from both control and IBD tissue cultures (Figure 6) do suggest a differential role of IFN-1 on non-T-cells, although the small tissue samples available precluded further investigation of this issue. Indeed, an early study on the role of interferon on lamina propria mononuclear cells (LPMCs) suggested that, in regard to cytotoxicity, LPMCs from IBD patients can be relatively hyporesponsive to exogenous interferon. ${ }^{28}$

Several of our observations indicate altered IFN-1 responsiveness and STAT1 phosphorylation of gut T-cells in IBD. For this analysis, we focused primarily on tissue sampled from macroscopically and histologically non-inflamed areas of intestine in order to identify underlying immunoregulatory changes that might otherwise be masked by ongoing inflammatory processes. The frequency of PSTAT1 + CD $4+$ T-cells detected was higher in non-inflamed IBD colon than in control tissue both in unstimulated cultures and after exposure to IFN-1 in vitro. This difference was specific for STAT1 in the intestinal mucosa, since we were unable to identify any modulation of alternative IFN-1-sensitive STATs or detect any changes in T-cells derived from the blood. Indeed, we were also unable to induce phosphorylation of STAT1 in control T-cells upon incubation with the culture supernatant of IBD biopsies, suggesting that enhanced pSTAT1 expression by IBD T-cells is an intrinsic property of these cells rather than being conferred by a soluble factor.

Previous studies have typically focused on analyzing STAT expression in whole samples of inflamed IBD tissue (containing numerous different cell types). Instead, we have for the first time used a novel phosflow-based method to measure the phosphorylated forms of STAT proteins at the single-cell level in distinct subsets of human leukocytes in the absence of overt mucosal inflammation. Using this approach, we observed an increased frequency of pSTAT1 + intestinal CD4 + T-cells in tissue from IBD patients, consistent with a previously shown trend for increased pSTAT1 levels in whole-tissue homogenates, and increases in total STAT1 protein identified in CD4 + lamina propria cells. ${ }^{29}$ However, we did not observe the previously reported increases in STAT3 expression and phosphorylation in $\mathrm{IBD},{ }^{29,30}$ which may reflect cell-type specific differences or could perhaps be attributed to our approach of analyzing non-inflamed tissue.

In addition to increased expression of pSTAT1, intestinal CD4 + T-cells from non-inflamed IBD intestinal tissue were significantly more responsive to IFN-1 stimulation as assessed by induction of ISG expression. Given the direct relationship between exposure to IFN-1 and expression of this gene set, this observation again suggests direct effects of IFN-1 on the T-cell population. SOCS1 is a major regulator of STAT1 signaling in $\mathrm{T}$-cells, and conditional $\mathrm{KO}$ of Socs 1 in murine T-cells has been shown to exacerbate colitis. ${ }^{31}$ SOCS1 is induced by IFN-1 and acts via numerous mechanisms as part of a negative feedback loop in IFN-1-STAT1 signaling. ${ }^{32}$ In our study, there was a strong trend toward reduced SOCS1 expression in intestinal T-cells freshly isolated from non-inflamed IBD tissue; we observed a corresponding increase in total STAT1 levels in these cells. It is therefore likely that reduced SOCS1 control of STAT1 signaling contributes to increased IFN-1 sensitivity of gut T-cells in IBD. Consistent with this altered responsiveness to IFN-1, we further observed that neutralization of IFN $\beta$ during IBD biopsy culture increased the frequency of derivative T-cells producing IFN $\gamma$, tumor necrosis factor- $\alpha$ or IL-17A. These data suggest that the non-inflamed IBD mucosa represents a modified signaling environment in which endogenous IFN $\beta$ acts to restrain production of pro-inflammatory cytokines rather than promoting IL-10 expression, perhaps to prevent the development of overt inflammation. We also detected a strong trend toward reduced ISG expression by gut T-cells when measured directly after isolation from IBD tissues compared with control tissue. Based on these observations, we propose that in IBD tissue the constituent T-cells exhibit altered responsiveness to endogenous IFN $\beta$ and other subsequent 
environmental signals, which could in part explain the seemingly conflicting early data on the role these cytokines play in IBD.

While the efficacy of IFN-1 treatment in IBD has been largely disappointing, ${ }^{20}$ there have been promising early results from a phase 1 trial of UC patients treated with the IFN-1-inducing TLR9 agonist DIMS0150. ${ }^{33}$ More recently, it has been demonstrated that patients with either UC or Crohn's disease have a distinct "virobiota" compared with household controls, ${ }^{34}$ suggesting that IFN-1 may be an important factor in the complex interactions between viruses, bacteria, and the host immune system during the pathogenesis of IBD.

In summary, this study demonstrates that human colon constitutively expresses IFN $\beta$, which has a selective STAT1dependent pro-regulatory effect on cytokine production by intestinal T-cells. This finding is consistent with mouse models in which IFN-1 signaling via STAT1 inhibits IFN $\gamma$ production. ${ }^{35}$ Our observations are also in agreement with the concept that the balance of STAT proteins and their activation determines the outcome of subsequent exposure to IFN-1 and other local triggers. Specifically, in this view IFN-1 maintains a basal repertoire STAT expression that forms a platform for specific T-cell responses. ${ }^{6}$

This IFN-1-shaped level of T-cell responsiveness would be an important determinant of local immune responses against viruses and other pathogens in the human intestine while preventing an excessive inflammatory response.

\section{METHODS}

Patient samples and cell isolation. Intestinal tissues were collected from patients with IBD during endoscopy or surgery, and control tissues were collected from subjects who were undergoing follow-up because of rectal bleeding, altered bowel habits, or cancer. The diagnosis for each patient was made using clinical parameters, radiographic studies, and histological criteria. All tissue and blood donors gave informed written consent, and the study was approved by the local research ethics committee. Patient information is presented in Supplementary Information.

Cells were extracted from intestinal tissue by enzymatic digestion or "walk-out" culture as previously described. ${ }^{36-38}$ Briefly, the tissue was treated with $1 \mathrm{mmoll}^{-1}$ dithiothreitol (Sigma-Aldrich, St Louis, $\mathrm{MO})$ to remove faeces and mucus, followed by $1 \mathrm{mmoll}^{-1}$ EDTA (Sigma-Aldrich) in Hank's balanced salt solution (Sigma-Aldrich) to remove epithelium. Digestion was by $1 \mathrm{mg} \mathrm{ml}^{-1}$ collagenase D (Roche Applied Science Penzberg, Upper Bavaria, Germany) in HEPESbuffered RPMI 1640 medium (Sigma-Aldrich) containing 2\% fetal calf serum (PAA, Little Chalfont, UK) and $20 \mu \mathrm{g} \mathrm{ml}^{-1}$ deoxyribonuclease I (Roche Applied Science). A lamina propria (LP) cell suspension was passed through a $40-\mu \mathrm{m}$ cell strainer. Alternatively, biopsies were cultured for "walk-out" following EDTA processing. The denuded tissue was placed in $0.5 \mathrm{ml}$ complete medium (RPMI1640 Dutch Modification supplemented with $2 \mathrm{M} \mathrm{L}$-glutamine, $100 \mathrm{U} \mathrm{ml}^{-1}$ penicillin, $100 \mu \mathrm{g} \mathrm{ml}^{-1}$ streptomycin, $100 \mu \mathrm{g} \mathrm{ml}^{-1}$ gentamicin (all SigmaAldrich) and $10 \%$ heat-treated $\left(56^{\circ} \mathrm{C}\right.$ for $30 \mathrm{~min}$ ) fetal calf serum (PAA). Cultures were established in 24-well plates (Becton Dickinson, Franklin Lakes, NJ) at one biopsy in $0.5 \mathrm{ml}$ of medium per well and placed in a humidified incubator at $37^{\circ} \mathrm{C}$ with $5 \% \mathrm{CO}_{2}$ in air. In some experiments the medium was supplemented with IFN $\beta$ (Peprotech, Rocky Hill, NJ) at $1000 \mathrm{IU} \mathrm{ml}^{-1}$ or with an equal volume of the diluent phosphate-buffer saline as a control. Alternatively, human anti-IFN $\beta$ antibody or an isotype-matched mouse IgG1 control antibody (both from Biolegend, San Diego, CA) were added at $1 \mu \mathrm{g} \mathrm{ml}^{-1}$ (Biolegend). The biopsies were then discarded after $48 \mathrm{~h}$ culture and egressed cells collected from the remaining medium by centrifugation at $400 \mathrm{~g}$ for $5 \mathrm{~min}$. Typically, $80 \%$ of viable cells isolated from non-inflamed or control samples were $\mathrm{CD} 3+$, and $75 \%$ of these cells were $\mathrm{CD} 4+$. Viability was assessed using Live/Dead staining.

Peripheral blood mononuclear cells were obtained from healthy volunteer donors or patients by centrifugation over Ficoll-Paque PLUS (GE Healthcare, Little Chalfont, UK) at $650 \mathrm{~g}$ for $20 \mathrm{~min}$.

Antibody labeling and flow cytometry. Cells were incubated with monoclonal antibodies on ice for $15 \mathrm{~min}$ in $100 \mu \mathrm{l}$ FACS buffer (phosphate-buffered saline with $2 \% \mathrm{v} / \mathrm{v}$ fetal calf serum, $1 \mathrm{mmol} 1^{-1}$ EDTA and $0.2 \%$ sodium azide), washed by centrifugation, and fixed in $1 \%$ (wt/vol) paraformaldehyde. Cells were acquired on a FACSCanto II (BD Biosciences, Franklin Lakes, NJ), using CellQuest software (BD Biosciences) and analyzed using WinList 6.0 software (Verity, Topsham, ME). Positively stained cells were identified relative to isotype-matched control antibody staining. A full list of the monoclonal antibodies used is provided in Supplementary Materials.

Analysis of phosphorylated antigens by flow cytometry. A technique for measuring intracellular phosphorylated antigens in single cells was developed and optimized as part of this study. LPMCs isolated by walk-out were re-suspended in $100 \mu \mathrm{l}$ complete medium with gentamicin in $5 \mathrm{ml}$ FACS tubes and either left unstimulated or stimulated with $40,000 \mathrm{IU} \mathrm{ml}^{-1}$ IFN $\alpha 2 \mathrm{~A}$ (Peprotech), for $15 \mathrm{~min}$ in a $37^{\circ} \mathrm{C}$ water bath. The cells were then fixed by addition of an equal volume of $4 \% \mathrm{w} / \mathrm{v}$ paraformaldehyde for $10 \mathrm{~min}$ in a $37^{\circ} \mathrm{C}$ water bath.

The fixed cells were washed by centrifugation in phosphate-buffer saline and then incubated in $1 \mathrm{ml}$ of $70 \%$ cold methanol (VWR, Radnor, PA) on ice for $30 \mathrm{~min}$ for permeabilization. The cells were washed twice in FACS buffer and then re-suspended in $100 \mu \mathrm{l}$ FACS buffer for simultaneous cell surface and intracellular antibody labeling. Cells were labeled as appropriate and incubated for 30-60 $\mathrm{min}$ at room temperature. After one further wash with FACS buffer the cells were re-suspended in $300 \mu \mathrm{l}$ of FACS buffer and analyzed by flow cytometry as described.

Stimulation of T-cells for cytokine analysis. LPMCs were cultured in $200 \mu \mathrm{l}$ complete medium in 96-well plates (Beckton Dickinson), and low endotoxin azide free anti-CD3 and anti-CD28 antibodies (Biolegend) were added each at a final concentration of $1 \mu \mathrm{g} \mathrm{ml}^{-1}$. The plates were put in a humidified incubator at $37^{\circ} \mathrm{C}$ with $5 \% \mathrm{CO}_{2}$ in air for $20 \mathrm{~h}$. After $20 \mathrm{~h}$ the cells were collected into $5 \mathrm{ml} \mathrm{FACS}$ tubes, and the Golgi-blocking agent monensin (VWR) was added at $3 \mu \mathrm{M}$ final concentration and the tube contents mixed thoroughly. The FACS tubes were replaced in the incubator for $4 \mathrm{~h}$. Cells were then collected and processed by flow cytometry after centrifugation and supernatants were stored at $-80^{\circ} \mathrm{C}$.

Isolation of intestinal or peripheral T-cells by flow sorting. LPMCs, collected by walk-out technique, were washed by addition of excess FACS buffer, centrifugation at $400 \mathrm{~g}$ for $5 \mathrm{~min}$ and discarding of supernatant. The cells were then resuspended into $100 \mu \mathrm{l}$ filter sterilized $(0.2 \mu \mathrm{m})$, modified FACS buffer (lacking sodium azide), and incubated with relevant monoclonal antibodies for $15 \mathrm{~min}$ on ice protected from light. Cell populations within LPCs were isolated by flow-sorting using a FACS Aria cytometer (Becton Dickinson). In order to sort only viable T-cells, the agent 7 -amino-actinomycin D (Biolegend) was used in combination with $\mathrm{CD}^{+}$labeling.

Quantitative real-time polymerase chain reaction. RNA extraction and reverse transcription were performed using the RNeasy Micro Kit and QuantiTect Reverse Transcription Kit, respectively. Quantitative real-time PCR was performed using the QuantiFast SYBR Green PCR Kit (all from Qiagen Venlo, Limburg, Netherlands) on a 7500 RealTime PCR System (Applied Biosystems, Foster City, CA). Full primers are listed in Supplementary Materials. Expression data were normalized to GAPDH using the $2^{-\Delta \mathrm{Ct}}$ method. 
Multiplex ELISA. Measurement of tumor necrosis factor- $\alpha$, IL-10, IFN $\gamma$, and IL-17A in culture supernatants was performed using the FlowCytomix Multiplex Kit (eBioscience, San Diego, CA) according to the manufacturer's instructions. Data were acquired on a Canto II Flow Cytometer (BD Bioscience), set up as recommended by the kit manufacturer and data analyzed using FlowCytomixPro (eBioscience) software.

Immunohistochemistry. Colonic biopsies were snap frozen in liquid nitrogen. Slides were prepared by the pathology service at the Royal London Hospital (Barts Health, London, UK). Slides were fixed with addition of $1 \mathrm{ml}$ of paraformaldehyde $4 \% .1 \mathrm{ml}$ blocking serum (5\% bovine serum albumin, $0.1 \%$ Tween in phosphate-buffer saline) was added followed by addition of primary antibodies. The antibodies used were as shown in Supplementary Materials. Secondary antibodies were applied: AF555 goat anti-mouse IgG (Invitrogen, Waltham, MA) and/or AF488 donkey anti-rabbit IgG (Invitrogen). Mounting medium was with DAPI (Vectashield, Vector Laboratories, Burlingame, CA).

Analysis was performed with a Research Systems Microscope BX61 (Olympus, Tokyo, Japan), with digital image capture at $\times 100$ (standard) or $\times 200$ (high power) using the software system SmartCapture v3.0 (Digital Scientific, Cambridge, UK).

Statistics. Statistical analyses were performed using SigmaStat 3.5 (Systat Software, San Jose, CA). Comparisons between two groups of paired, normally and non-normally distributed data were analyzed using Paired $t$-test, Student's $t$-test and Signed rank test, respectively. Data sets of more than two groups were compared by analysis of variance with pairwise comparisons by Holm-Sidak test. $P<0.05$ was regarded as statistically significant.

SUPPLEMENTARY MATERIAL is linked to the online version of the paper at http://www.nature.com/mi

\section{ACKNOWLEDGMENTS}

This work was supported by a research fellowship grant from the Crohn's and Colitis in Childhood Research Association (CICRA) and a small project grant from Crohn's and Colitis UK (CCUK). We acknowledge Professor lan Sanderson, who helped with the initial design of this work and provided important support throughout. We also thank Dr Gary Warne for his advice and assistance in the use of the sorting by flow cytometry. We also thank $\mathrm{Dr}$ Raj Lahiri and Professor Graham Foster for the kind gift of the primers for the ISGs $\left(2^{\prime} 5^{\prime}\right.$ OAS and MxA).

\section{AUTHOR CONTRIBUTIONS}

E.M.G. designed, performed, and analyzed the experiments and wrote the manuscript. A.J.S. designed experiments and wrote the manuscript. T.J.S. and N.E.M. performed experiments and wrote the manuscript. M.P. and J.L. performed and analyzed experiments. J.O.L. and T.T.M. designed experiments and assisted in the manuscript preparation.

\section{DISCLOSURE}

The authors declared no conflict of interest.

(c) 2017 Society for Mucosal Immunology

\section{REFERENCES}

1. Neurath, M.F. Cytokines in inflammatory bowel disease. Nat. Rev. Immunol. 14, 329-342 (2014).

2. O'Shea, J. \& Paul, W. Mechanisms underlying lineage commitment and plasticity of helper CD4 + T cells. Science 327, 1098-1102 (2010).

3. Strober, W. \& Fuss, I.J. Proinflammatory cytokines in the pathogenesis of inflammatory bowel diseases. Gastroenterology 140, 1756-1767 (2011).

4. Laouar, A. et al. CD70 + antigen-presenting cells control the proliferation and differentiation of T cells in the intestinal mucosa. Nat. Immunol. 6, 698-706 (2005).

5. Tough, D.F. Modulation of T-cell function by type I interferon. Immunol. Cell Biol. 90, 492-497 (2012).
6. Gough, D.J., Messina, N.L., Clarke, C.J., Johnstone, R.W. \& Levy, D.E. Constitutive type I interferon modulates homeostatic balance through tonic signaling. Immunity 36, 166-174 (2012).

7. Nguyen, K.B. et al. Critical role for STAT4 activation by type 1 interferons in the interferon-gamma response to viral infection. Science 297, 2063-2066 (2002).

8. Teijaro, J.R. et al. Persistent LCMV infection is controlled by blockade of type I interferon signaling. Science 340, 207-211 (2013).

9. Wilson, E.B. et al. Blockade of chronic type I interferon signaling to control persistent LCMV infection. Science 340, 202-207 (2013).

10. Odorizzi, P.M. \& Wherry, E.J. Immunology. An interferon paradox. Science 340, 155-156 (2013).

11. Cho, H. \& Kelsall, B.L. The role of type I interferons in intestinal infection, homeostasis, and inflammation. Immunol. Rev. 260, 145-167 (2014).

12. McFarland, A.P. et al. Localized delivery of interferon- $\beta$ by Lactobacillus exacerbates experimental colitis. PLoS One 6, e16967 (2011).

13. Abe, K. et al. Conventional dendritic cells regulate the outcome of colonic inflammation independently of T cells. Proc. Natl. Acad. Sci. USA 104, 17022-17027 (2007).

14. Bleich, A. et al. CpG motifs of bacterial DNA exert protective effects in mouse models of IBD by antigen-independent tolerance induction. Gastroenterology 136, 278-287 (2009).

15. Kole, A. et al. Type I IFNs regulate effector and regulatory $T$ cell accumulation and anti-inflammatory cytokine production during $T$ cellmediated colitis. J. Immunol. 191, 2771-2779 (2013).

16. Lee, S.E. et al. Type I interferons maintain foxp3 expression and T-regulatory cell functions under inflammatory conditions in mice. Gastroenterology 143, 145-154 (2012).

17. Radulovic, K. et al. CD69 regulates type I IFN-induced tolerogenic signals to mucosal CD4 T cells that attenuate their colitogenic potential. J. Immunol. 188, 2001-2013 (2012).

18. Axtell, R.C. et al. T helper type 1 and 17 cells determine efficacy of interferon-beta in multiple sclerosis and experimental encephalomyelitis. Nat. Med. 16, 406-412 (2010).

19. Jostins, L. et al. Host-microbe interactions have shaped the genetic architecture of inflammatory bowel disease. Nature 491, 119-124 (2012).

20. Seow, C.H., Benchimol, E.I., Griffiths, A.M. \& Steinhart, A.H. Type I interferons for induction of remission in ulcerative colitis. Cochrane Database Syst. Rev. CD006790, 16 (2008).

21. Teles, R.M. et al. Type I interferon suppresses type II interferon-triggered human anti-mycobacterial responses. Science 339, 1448-1453 (2013).

22. Afkarian, M. et al. T-bet is a STAT1-induced regulator of IL-12R expression in naîve CD4 + T cells. Nat. Immunol. 3, 549-557 (2002).

23. Neurath, M.F. et al. The transcription factor T-bet regulates mucosal T cell activation in experimental colitis and Crohn's disease. J. Exp. Med. 195, 1129-1143 (2002).

24. Kelsen, J. et al. FoxP3 $(+) \mathrm{CD} 4(+) \mathrm{CD} 25(+) \mathrm{T}$ cells with regulatory properties can be cultured from colonic mucosa of patients with Crohn's disease. Clin. Exp. Immunol. 141, 549-557 (2005).

25. Hofmann, C. et al. T cell-dependent protective effects of CpG motifs of bacterial DNA in experimental colitis are mediated by CD11c + dendritic cells. Gut 59, 1347-1354 (2010).

26. Wang, J., loan-Facsinay, A., van der Voort, E.I., Huizinga, T.W. \& Toes, R.E. Transient expression of FOXP3 in human activated nonregulatory CD4 + T cells. Eur. J. Immunol. 37, 129-138 (2007).

27. Metidji, A. et al. IFN- $\alpha / \beta$ receptor signaling promotes regulatory $T$ cell development and function under stress conditions. J. Immunol. 194, 4265-4276 (2015).

28. MacDermott, R.P., Bragdon, M.J., Kodner, I.J. \& Bertovich, M.J. Deficient cell-mediated cytotoxicity and hyporesponsiveness to interferon and mitogenic lectin activation by inflammatory bowel disease peripheral blood and intestinal mononuclear cells. Gastroenterology 90, 6-11 (1986).

29. Mudter, J. et al. Activation pattern of signal transducers and activators of transcription (STAT) factors in inflammatory bowel diseases. Am. J. Gastroenterol. 100, 64-72 (2005).

30. Lovato, P. et al. Constitutive STAT3 activation in intestinal $T$ cells from patients with Crohn's disease. J. Biol. Chem. 278, 16777-16781 (2003).

31. Horino, J. et al. Suppressor of cytokine signaling-1 ameliorates dextran sulfate sodium-induced colitis in mice. Int. Immunol. 20, 753-762 (2008). 
32. Palmer, D.C. \& Restifo, N.P. Suppressors of cytokine signaling (SOCS) in T cell differentiation, maturation, and function. Trends Immunol. 30, 592-602 (2009).

33. Musch, E. et al. Topical treatment with the Toll-like receptor agonist DIMS0150 has potential for lasting relief of symptoms in patients with chronic active ulcerative colitis by restoring glucocorticoid sensitivity. Inflamm. Bowel Dis. 19, 283-292 (2013).

34. Norman, J.M. et al. Disease-specific alterations in the enteric virome in inflammatory bowel disease. Cell 160, 447-460 (2015).
35. Nguyen, K.B. etal. Interferon alpha/beta-mediated inhibition and promotion of interferon gamma: STAT1 resolves a paradox. Nat. Immunol. 1, 70-76 (2000).

36. Mahida, Y.R. et al. Migration of human intestinal lamina propria lymphocytes, macrophages and eosinophils following the loss of surface epithelial cells. Clin. Exp. Immunol. 109, 377-386 (1997).

37. Hart, A. et al. Characteristics of intestinal dendritic cells in inflammatory bowel diseases. Gastroenterology 129, 50-65 (2005).

38. Bell, S.J. et al. Migration and maturation of human colonic dendritic cells. J. Immunol. 166, 4958-4967 (2001). 